\title{
Des animaux dans la littérature : du proche et du lointain
}

Nigel LEZAMA, Université Brock, Joëlle PAPILLON, Université McMaster, et Rocky PENATE, Université de Waterloo

Dans 1'«Apologie de Raimond Sebond», essai écrit ostensiblement pour défendre le savant éponyme dont les écrits - qui auraient fait l'objet d'une certaine controverse - cherchaient à réconcilier foi chrétienne et science, Michel de Montaigne constate l'étendue de ce que l'être humain ignore des animaux : «Comment cognoit il, par l'effort de son intelligence, les branles internes et secrets des animaux ? par quelle comparaison d'eux à nous conclud il la bestise qu'il leur attribue?»(452). Pour quiconque s'est déjà interrogé sur la possibilité de dire et écrire fidèlement les animaux, cette ignorance est l'évidence même. Il s'agit d'un référent fuyant, difficile sinon impossible à saisir - l'animal textuel est toujours un succédané d'animal. Ce sont des êtres à la fois familiers et radicalement autres, apprivoisables et insaisissables, nous «[donnant] à penser cette altérité absolue du voisin ou du prochain » (Derrida 28), d'où le foisonnement d'animaux transfigurés dans nos mythes, légendes, poèmes, romans et autres

productions culturelles, du Roman de Renart à L'histoire de Pi, pour se limiter à l'époque moderne et à la sphère occidentale. La grande majorité de ces représentations réduisent les animaux au symbolique, à un faire-valoir de l'humanité. C'est que, pour citer une réflexion classique sur l'impossibilité de connaître à fond l'expérience animale - l'essai de Thomas Nagel sur la conscience des chauves-souris, «What is it like to be a bat? » (1974) -, tout se passe comme si on essayait de saisir le vol de la chauve-souris, qui se repère dans le monde grâce à un sonar, au moyen d'analogies basées sur nos cinq sens modestement développés.

Les tentatives de mieux connaître les animaux, de s'en rapprocher par le biais de la littérature sont-elles donc vouées à la futilité ? Les articles ici recueillis contestent une telle abdication du pouvoir littéraire. Portant sur des textes issus de diverses époques de l'histoire occidentale ainsi que sur la tradition orale autochtone, le présent dossier permettra aux lectrices et aux lecteurs de retracer le développement des idées que nous nous faisons sur les animaux depuis l'avènement de la modernité, laquelle repose en grande partie sur les barrières que nous établissons entre culture et nature, humain et animal. Comme l'explique Bruno Latour dans sa critique du concept de la modernité : 
Modernity is often defined in terms of humanism, either as a way of saluting the birth of «man» or as a way of announcing his death. But this habit itself is modern, because it remains asymmetrical. It overlooks the simultaneous birth of « nonhumanity »- things, or objects, or beasts - and the equally strange beginning of a crossed-out God, relegated to the sidelines. Modernity arises first from the conjoined creation of those three entities, and then from the masking of the conjoined birth and the separate treatment of the three communities while, underneath, hybrids continue to multiply as an effect of this separate treatment. (13)

Pour commencer à rectifier l'asymétrie constitutive de la modernité dont traite Latour, il faudrait prêter attention un tant soit peu à toute cette vaste non-humanité, ce que fait le domaine foncièrement interdisciplinaire qu'on appelle études animales. Les idées de Latour font écho à celles de Donna Haraway, qui elle aussi affirme des réalités essentiellement hybrides : «By the late twentieth century, our time, a mythic time, we are all chimeras, theorized and fabricated hybrids of machine and organism; in short, we are cyborgs », c'est-à-dire des créatures « simultaneously animal and machine, who populate worlds ambiguously natural and crafted » (n.p.). C'est dans la perspective de l'hybridité des êtres modernes, inéluctablement entremêlés dans des processus culturels comme naturels, qu'il nous semble pertinent d'étudier la mise en discours des vies animales. C'est ainsi que la littérature peut se révéler apte à rendre compte de l'être humain, des animaux et des croisements qui conditionnent leur coexistence.

En effet, chacun des articles réunis dans ce dossier - qu'il traite de poésie, de romans et récits, ou d'écrits plutôt philosophiques, voire théoriques - met en relief le trouble qu'occasionne toujours la question du rapport entre l'humain et l'animal : trouble langagier, trouble esthétique, trouble social, trouble éthique, entre autres. Force est de constater que l'insuffisance du logos à rendre compte du rapport humain-animal déclenche aussi bien qu'il ne fait tarir l'écriture. La littérature peut se lire comme une histoire du rapprochement et de l'éloignement alternatifs - et souvent simultanés - entre êtres humains et animaux.

Certes, en parcourant ces articles, il devient clair que l'on ne peut guère parler d'un progrès des relations entre humains et animaux ni de leurs représentations. Sous l'apparence de «progrès », une dénégation fondamentale conditionne notre perception des autres animaux. Cette dénégation est souvent qualifiée de «cartésienne» mais précède et dépasse le grand rationaliste ; celui-ci se serait démarqué surtout par sa représentation des animaux en automates, dépourvus, entre autres, de la possibilité de (nous) répondre, représentation qui vient clore un débat vieux de plusieurs millénaires : 
Pourquoi cette argumentation bien connue a-t-elle choqué, et n'en finit pas de choquer le bon sens partagé qu'elle expose et traduit pourtant? C'est qu'elle venait interrompre de longs débats historiques [...]. Une interminable conversation avait déjà engagé tous ceux qui, de Plutarque à Porphyre et à Montaigne [...] n'avaient cessé d'agiter en tous sens les questions que Descartes vient enfin trancher, de son pas cavalier de «cavalier français », avec autant d'autorité. (Derrida 118)

Néanmoins, comme on le voit dans l'article de Swann Paradis, le débat se poursuivra, mettant cartésiens en opposition aux anticartésiens; au dix-neuvième siècle, le débat résulte en la fondation de nombreux mouvements pour la protection des animaux; aujourd'hui, il est médiatisé autant que possible. Cependant, les tueries industrielles des animaux continuent aussi sinon plus intensément qu'auparavant. Il y a lieu de se demander : qu'y peut la littérature ? Pratique foncièrement subjective, elle peut, tout au moins, nous rappeler la présence animale. Que nous en soyons conscients ou non, les animaux font partie intégrante de notre monde ; comme l'illustrent les articles dans ce numéro, les animaux nous aident à nous exprimer, à nous représenter les relations de pouvoir, à penser notre « âme », à affronter la mort... Bien entendu, jusqu'à présent, les animaux nous accompagnent généralement à leur détriment; nous nous risquons à espérer que la littérature puisse contribuer à une amélioration de leur sort. Ce dossier se veut donc une contribution originale non seulement au développement des études animales dans le domaine des lettres, mais aussi un aperçu de ce que l'accompagnement animal a déjà été au sein de la vie humaine et de ce qu'il pourrait encore devenir.

Nous tenons à souligner le fait qu'à l'exception notable de Richard Lefebvre, nous avons interrogé des littératures issues uniquement de cultures occidentales. L'article de Lefebvre nous rappelle qu'il existe d'autres façons de penser la rencontre entre humain et animal et nous fait espérer que ces possibilités autres pourront éventuellement remettre en question et enrichir les idées qui président au rapport humain-animal dans les sociétés occidentales. Dans son étude sur la représentation des animaux dans le roman qui a fait date, Lucile Desblache constatait que les littératures postcoloniales, par exemple, «explorent des modèles qui ne sont pas fondés sur une pensée mécaniste et prennent du recul vis-à-vis de la tradition humaniste qui place l'homme au sommet de la hiérarchie des êtres vivants »(21). Voilà une piste prometteuse, à condition de l'emprunter de façon critique et de ne pas reconvoquer de vieilles idées sur le primitivisme des cultures non occidentales. Il nous semble au contraire qu'il faudrait avancer sous l'égide de l'hybride, ce produit du contact et de la circulation entre diverses cultures et natures. 


\section{Contributions au numéro}

Face à l'humain, l'animal apparaît polymorphe. Son altérité réconforte. Son analogie perturbe. Pour certains, les animaux sont source du réconfort offert par ce qui nous est familier, tandis que chez d'autres encore c'est leur différence qui attire. La représentation littéraire de la figure animale est un angle polysémique grâce auquel la littérature de tous les siècles et provenant de maintes régions géographiques peuvent se rapprocher. Quelle que soit la mise en scène des animaux adoptée par le texte littéraire, il y a une relation éthique, philosophique et politique entre le sujet écrivant et le sujet représenté qui s'y décèle. Ce dossier sur l'affirmation et la dénégation de l'animalité se propose de mettre en lumière les liens et les écarts entre les animaux non humains et ceux qui vivent en leur proximité. Le premier volet du dossier se compose d'articles traitant de la distance, proche ou éloignée, qui sépare l'humain et l'animal. Dans le deuxième volet, c'est la fonction littéraire de l'animal, l'animal comme figure rhétorique, qui sera mise en lumière.

Nous abordons la question avec la polémique de l'âge classique sur la question de la distinction homme-animal, voire la distinction âme-corps, qu'Alex Bellemare investigue de façon originale dans son article qui porte sur l'animalité et la monstruosité dans deux romans libertins, L'autre monde de Cyrano de Bergerac et La terre australe connue de Gabriel de Foigny. Le roman de ce dernier évoque le «non-lieu» austral dans le récit d'un personnage hermaphrodite qui brandit le jugement d'animalité pour appuyer son appartenance à la race humaine. Cyrano, de son côté, raconte un voyage extraterrestre qui met en scène deux mondes, lunaire et solaire, peuplés d'êtres curieux et animaliers, dont l'existence inversera la primauté supposée de l'homme.

Abordant lui aussi la question de la primauté humaine face à l'animal, l'article de Swann Paradis privilégie trois moments épistémologiques dans la pensée philosophique sur l'animal. Lors du siècle des Lumières, il devient de plus en plus difficile de considérer la bête seulement sous son aspect mécanique. Paradis se penche sur le Père Bougeant qui élabore une pensée sur la perfectibilité animale ; sur Condillac qui octroie à l'animal une âme immatérielle, mais mortelle ; et, finalement, sur Charles Bonnet qui rapproche la bête de l'homme grâce à sa perception du jugement animal. Paradis met au jour la transformation nuancée de la conception religieuse de la bête (refusant l'existence d'une âme animale) à l'approche sensualiste qui pose un regard plus généreux sur les animaux. 
Dans un article qui analyse la mise en scène de deux personnages à l'animalité marquée dans Les mystères de Paris, Nigel Lezama examine la représentation littéraire à la lumière des discours social et juridique sur l'animal. Son travail révèle les tensions provenant du domaine juridique qui, après la Révolution, pose les jalons d'une pratique plus éthique envers l'animal. Ce regard éthique trouve son pendant dans la grande question sociale de la pauvreté et de la criminalité qui taraude les penseurs au dix-neuvième siècle.

Pour sa part, Joëlle Papillon se penche sur l'intimité qui peut exister entre humains et animaux dans deux œuvres récentes de l'écrivaine québécoise Élise Turcotte. Dans le monde turcottien, c'est grâce aux liens affectifs avec les animaux de compagnie que les narratrices trouvent le moyen de surmonter des tragédies personnelles. Cette relation d'appui dans la douleur mène à une identification de la part de l'humain avec la souffrance animale et illustre la possibilité de trouver un soutien moral inattendu pour remédier à un isolement humain pénible.

Se penchant sur la représentation des animaux chez Alphonse de Lamartine et Francis Ponge, Rocky Penate amorce la deuxième partie de notre dossier en arpentant les limites à l'expression de l'animal dans le discours poétique. L'animal a été l'objet du regard scientifique, mais invariablement dans un but anthropocentriste : grâce à l'animal, il serait possible de déceler le propre de l'humain. Penate met en lumière les similarités surprenantes qui rapprochent le poète romantique et son homologue moderne, similarités au niveau d'un traitement textuel réducteur qui valorise l'utilité de l'animal pour l'humain. Cet article insiste sur la possibilité d'une conduite plus éthique où l'humain mettrait en avant la dimension affective qu'inspire l'animal.

Nicolas Gauthier s'intéresse pour sa part au personnage canin dans le roman-feuilleton Les Mohicans de Paris d'Alexandre Dumas. Dans la représentation qu'en fait l'écrivain, Gauthier décèle un véritable brouillage de traits qui distinguent l'humain de l'animal. Grâce à une mise en scène anthropomorphisante, le chien constitue un rouage narratif indéniable. Cependant, l'animalité dans ce texte est également le moyen par lequel Dumas souligne la parenté entre l'humain et l'animal, ce qui lui permet d'avancer le projet politique du roman.

Dans un article qui porte sur la présence et la transformation du cliché «avoir d'autres chats à fouetter » dans trois romans de Nathalie Sarraute, Sarah Anthony décèle deux modalités antinomiques à la figuration animale, soit l'image animalière et la banalité zoologique. Chacune de ces figurations occupe une fonction particulière au sein du récit sarrautien, celle-ci servant à 
signaler le personnage qui refuse toute motivation psychologique tandis que celle-là représente l'expérience tropismique du personnage. Cet usage contradictoire de l'expression figée animalière met en relief le rôle de la figure animale dans la construction de la dominance humaine dans l'œuvre de Sarraute.

Nous terminons notre dossier avec l'article de Richard Lefebvre, qui élucide la fonction actancielle de l'animal dans le récit oral algonquien. De façon intéressante, Lefebvre souligne également une lacune dans la représentation de la communication animale qui a lieu lors de la transcription de ces récits, à l'origine oraux, par l'ethnographe exogène. L'auteur propose en l'occurrence que l'oralité et la performance de la personne qui conte, permettent à l'animal de préserver sa singularité.

Cette introduction ne serait ni complète ni tout à fait juste si nous ne mentionnions combien nous sommes redevables à notre amie la professeure Angela Cozea, dont la réflexion sur les animaux précède et donne lieu à la nôtre.

\section{Bibliographie}

Desblache, Lucile. La plume des bêtes. Les animaux dans le roman. Paris : Harmattan, 2011.

Derrida, Jacques. L'animal que donc je suis. Paris : Galilée, 2006.

Haraway, Donna. «A Cyborg Manifesto. Science, Technology, and Socialist-Feminism in the Late Twentieth Century ». Simians, Cyborgs and Women : the Reinvention of Nature. New York : Routledge, (1991). 149-181.n. pag. Web. $7 \quad$ septembre 2015. <http://www.egs.edu/faculty/donna-haraway/articles/donna-haraway-a-cyborg-manifesto/>

Latour, Bruno. We Have Never Been Modern. Tr. Catherine Porter. Cambridge : Harvard UP, 1993.

Montaigne, Michel de. Les Essais. Livre II. Pierre Villey, dir. Paris : Quadridge/PUF, 1992 [1588]. 